\title{
A small, low-grade rectal neuroendocrine tumor with lateral pelvic lymph node metastasis: a case report
}

\author{
Seonhui Shin ${ }^{1}$, Young-In Maeng ${ }^{2}$, Seyun Jung ${ }^{1}$, Chun-Seok Yang ${ }^{1}$ \\ Departments of ${ }^{1}$ Colorectal Surgery and ${ }^{2}$ Pathology, Daegu Catholic University Medical Center, Daegu Catholic University School of \\ Medicine, Daegu, Korea
}

Rectal neuroendocrine tumors (NETs) are typically small lesions that are confined to the submucosa and have favorable behavior at the time of diagnosis. Local endoscopic or surgical resection is recommended because lymph node metastasis is very rare. In this report, we present the case of a 36-year-old male presenting with an incidentally found rectal mass during screening colonoscopy. Pathologic examination of the primary tumor revealed a 9-mm grade 1 NET with submucosal invasion and no significant aggressive factors except for central ulceration. However, radiologic studies revealed a suspected $2.6-\mathrm{cm}$ mesorectal lymph node metastasis and multiple left internal iliac lymph node metastases. We performed laparoscopic intersphincteric resection with left lateral pelvic lymph node dissection. The final pathologic report revealed a metastatic lymph node with low grade, low mitotic count, and low Ki-67 index. We describe an overview of lymph node metastasis of rectal NETs focusing on lateral pelvic lymph node metastasis.

Keywords: Neuroendocrine tumors; Carcinoid tumor; Lymph node excision; Lymphatic metastasis; Case report

\section{INTRODUCTION}

Neuroendocrine tumors (NETs) are rare, slow-growing, and heterogeneous neoplasms that can arise from the diffuse neuroendocrine system, the most common of which are in the gastrointestinal (GI) tract, the bronchopulmonary system, thymus, and pancreas [1]. The most common location of NETs in the GI tract among patients in the United States is the small intestine (38\%), followed by the rectum $(34.4 \%)$, colon (16.2\%), stomach (10.6\%), and other unknown sites (0.75\%), according to an analysis of the Surveillance Epidemiology End Results database [2]. In contrast, the rectum (48\%) is the most frequent location of NETs in the GI tract of patients in Korea [3]. Their incidence has been steadily

Received: Oct 12, 2021 - Revised: Dec 20, 2021 - Accepted: Dec 24, 2021

Correspondence to: Chun-Seok Yang, M.D.

Department of Colorectal Surgery, Daegu Catholic University Medical Center, Daegu Catholic University School of Medicine, 33 Duryugongwon-ro 17-gil, Nam-gu, Daegu 42472, Korea

Tel: +82-53-650-4065, Fax: +82-53-624-7185

E-mail: gsyangcs@gmail.com

ORCID: https://orcid.org/0000-0002-5527-6819

(c) 2022 The Korean Society of Coloproctology

This is an open-access article distributed under the terms of the Creative Commons Attribution NonCommercial License (https://creativecommons.org/licenses/by-nc/4.0) which permits unrestricted noncommercial use, distribution, and reproduction in any medium, provided the original work is properly cited. increasing over the past few decades regardless of country or continent. The variation in primary tumor sites could be attributed to the development of better diagnostic tools and improved knowledge, and these lead to increased incidental detection of asymptomatic rectal NETs [4].

Most rectal NETs are diagnosed incidentally during screening colonoscopy. Approximately $80 \%$ of them are $10 \mathrm{~mm}$ or less in size, and up to $90 \%$ of tumors are confined within the submucosal layer at diagnosis $[5,6]$. For small $(<10 \mathrm{~mm})$ and incidental lesions, local resection by endoscopy or transanal excision is recommended if there is no evidence of invasion beyond the submucosa or presence for regional disease. The reason why local resection is considered sufficient is that lymph node metastasis is very rare, appearing in only $2.5 \%$ of tumors less than $10 \mathrm{~mm}$ [7]. However, we intend to report on metastasis of the lateral pelvic lymph nodes (LPLN) found in a patient with a NET less than $1 \mathrm{~cm}$ in size that did not invade the muscularis properia. There are very few case reports on LPLN metastasis from rectal NETs, especially in the English language. In this report, we present the case of a patient with small rectal NETs with LPLN metastases who underwent laparoscopic intersphincteric resection with LPLN dissection (LPLD). 


\section{CASE REPORT}

A 36-year-old male patient presenting with an incidentally found rectal mass (Fig. 1) during screening colonoscopy was referred to our institute. He had undergone transanal excision of the rectal mass at another hospital 1 month ago. A biopsy specimen of the primary tumor resected by another hospital suggested a $9 \times 5-\mathrm{mm}$ grade 1 NET. The tumor invaded $5 \mathrm{~mm}$ of the submucosal layer with a free resection margin, but lymphovascular and perineural invasion were absent. No increased atypia of tumor cells or mitosis ( $<1$ mitosis per 10 high power fields) were found but there

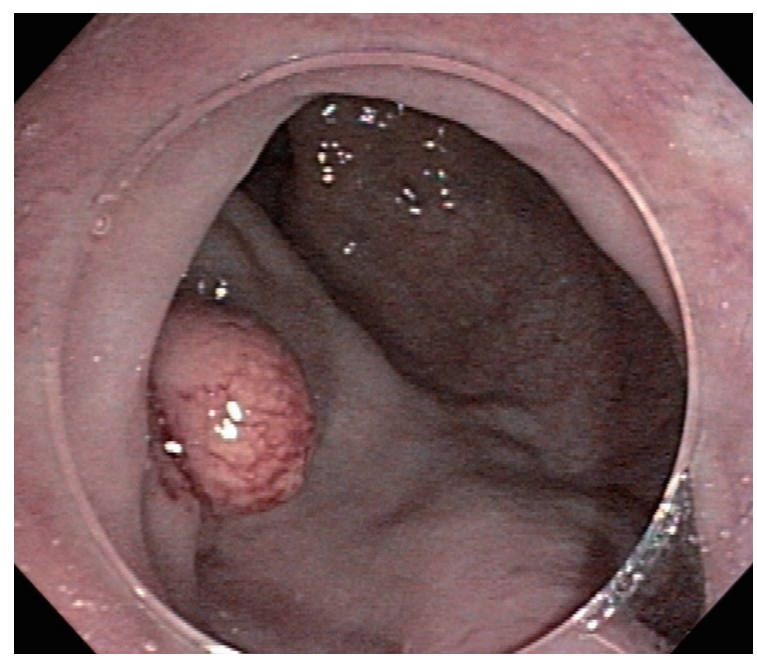

Fig. 1. Colonoscopic appearance of the primary tumor at the distal rectum.

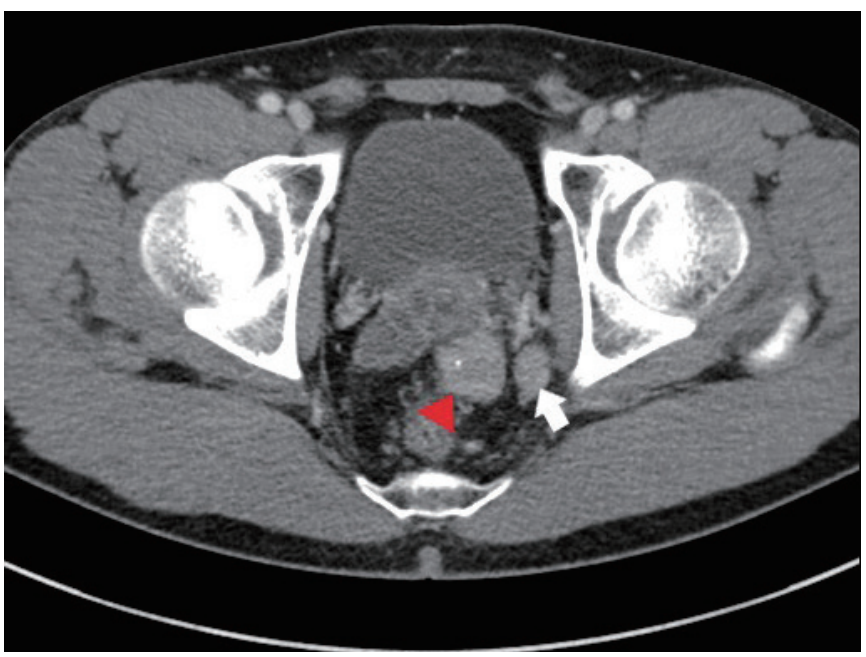

Fig. 2. Preoperative abdominopelvic computed tomography scan showing a $2.6-\mathrm{cm}$ enhancing mass (red arrowhead) in the left lateral rectal wall with left internal iliac lymph node enlargements (white arrow). was surface ulceration. In our hospital, abdominopelvic computed tomography (CT) (Fig. 2) showed a 2.6-cm sized enhancing mass in the left lateral rectal wall with multiple left internal iliac lymph node enlargements, the largest of which reached $2.2 \mathrm{~cm}$. We thought that the enhancing mass at the left lateral rectal wall was from a remnant disease before performing sigmoidoscopy. However, sigmoidoscopy revealed only a postexcisional scar in the rectum, $3 \mathrm{~cm}$ above the anal verge. Positron emission tomography/CT scan using F-18 fluorodeoxyglucose (FDG) (Fig. 3) revealed an FDG uptake of 6.17 of maximum standardized uptake value (SUVmax) of the left lateral rectal mass and an FDG uptake of 4.51 of SUVmax of the internal iliac lymph nodes.

We performed laparoscopic intersphincteric resection, handsewn coloanal anastomosis, diverting ileostomy, and left LPLD. The procedures were performed using laparoscopic colonic mobilization with inferior mesenteric artery ligation. In the pelvic approach, a total mesorectal excision was performed up to the anal canal. At the anal approach, the distal resection line was placed below the previously excised scar and transanal dissection was performed toward the intraperitoneal space. The LPLD was started after the rectum was completely transected and moved from the pelvis into the abdominal cavity. A left LPLD was subsequently performed as follows: the left ureter and left hypogastric nerve were isolated and the lymph nodes were dissected from the bifurcation of the aorta extending to the common iliac area. The internal iliac vessels were subsequently cleared from the lymphatic tissue at a safe distance from the lateral side of the pelvic plexus. During dissection, the obturator nerve and vessels were identified medial to the external iliac vein and lateral to the superior vesical artery. Dissection was continued downward to the middle rectal artery area while removing all lymph nodes from the narrow

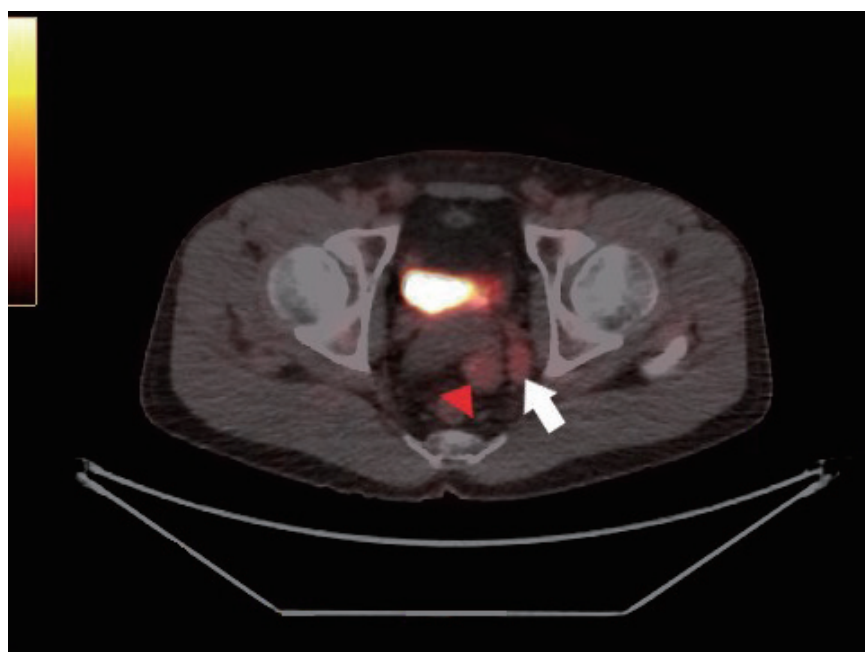

Fig. 3. Positron emission tomography/computed tomography scan revealed high F-18 fluorodeoxyglucose uptake in the left lateral mesorectum (red arrowhead) and left internal iliac area (white arrow). 
space between the terminal branches of the internal iliac vessels and the bony pelvis, and the space between those vessels and the pelvic nerves. The lymph nodes were collected in a plastic bag. Following completion of the pelvic lymph node dissection, only

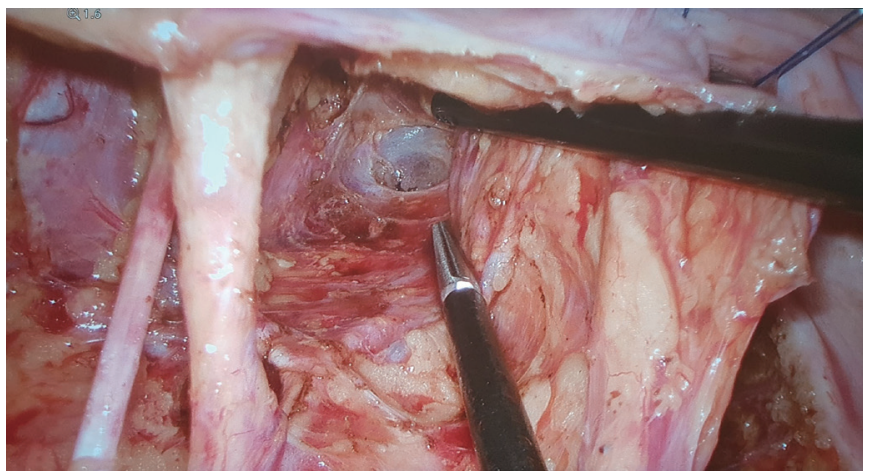

Fig. 4. Laparoscopic view after left lateral pelvic lymph node dissection. the external vessels, internal iliac vessels and their branches, the obturator nerve, and the pelvic plexus remained (Fig. 4). Finally, the specimen was removed from the anus and was reconstructed with a hand-sewn anastomosis.

On abdominal CT, an enhancing mass at the left lateral wall of the rectum was confirmed as a $3.5-\mathrm{cm}$ subserosal mass that appeared to be regional lymph node metastasis. Of the 9 harvested lymph nodes from the left pelvic sidewall, 4 metastasized lymph nodes were identified (Fig. 5). Pathological examination of the metastatic lymph nodes showed the tumor cells spreading in a rosette-like pattern with a Ki-67 index of 2.3\%. Immunohistochemical staining revealed the tumor cells to be positive for synaptophysin and CD56. The postoperative course was uneventful, and the patient was scheduled to continue to receive follow-up examinations regularly. Adjuvant therapy was not performed and diverting stoma closure was performed 2 months after the initial operation. The patient is doing well without recurrence 32 months after surgery.

The Institutional Review Board of Daegu Catholic University
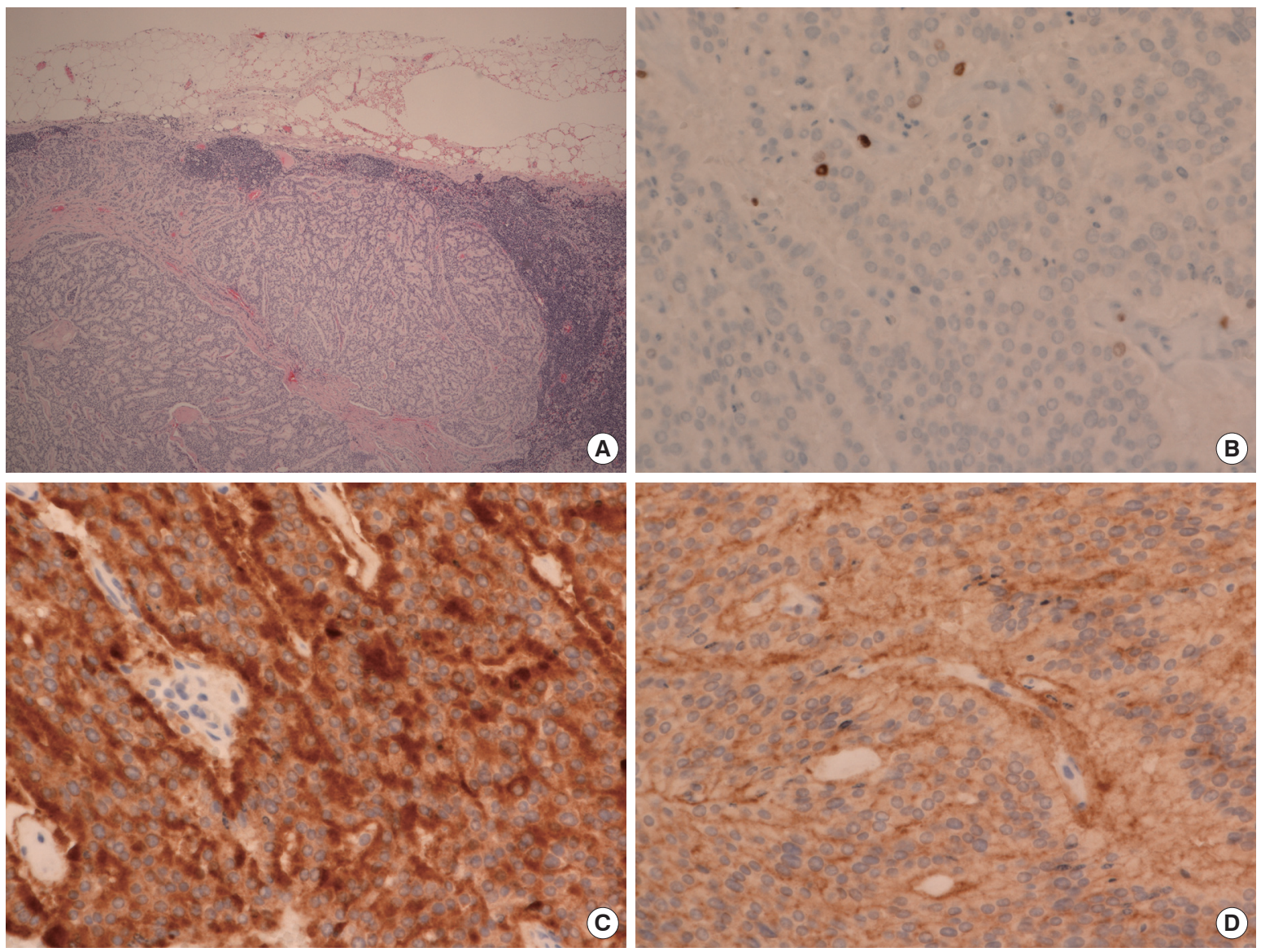

Fig. 5. Histopathological findings of the left internal iliac lymph node from the resected specimen showed metastatic tumor cells (A) spread in a rosette-like pattern $(\mathrm{H} \& \mathrm{E}, \times 200)$. (B) The Ki-67 index was 2.3\% ( $\times 400)$. Immunohistochemical staining for synaptophysin $(\mathrm{C})$ and CD56 (D) were positive $(\times 400)$. 
Hospital approved this case report (No. CR-21-168) and waived the informed consent requirement.

\section{DISCUSSION}

Rectal NETs are usually small lesions (mostly $<1 \mathrm{~cm}$ ) that are confined to the submucosa. Their histological malignancy is low to intermediate grade at the time of diagnosis. These rectal NETs usually underwent endoscopic resection initially because the possibility of lymph node metastasis was low or the pathologic diagnosis was not confirmed at detection. In the National Comprehensive Cancer Network guidelines, for small $(<1 \mathrm{~cm})$ and incidental lesions, complete endoscopic resection with negative margins may be sufficient. Meanwhile, any tumor of $>2 \mathrm{~cm}$ or with evidence of metastatic disease in any tumor size is recommended for radical resection [8]. In the European Neuroendocrine Tumor Society guidelines, patients with rectal NETs of $<1 \mathrm{~cm}$ with or without muscular invasion (T1 or T2) are recommended to undergo endoscopic resection or complete local resection [9]. However, in our case, the tumor was $9 \mathrm{~mm}$ in diameter, and the pathological tumor depth was limited to the submucosal layer but showed extensive metastasis to the mesorectal and LPLN.

The decision regarding the treatment modality for NETs depends on the possibility of lymph node metastasis. It depends on their size, grade of differentiation, muscularis propria involvement, lymphatic and vascular invasion, and tumor proliferative index. Tumor size is one of the most important prognostic factors, and Gleeson et al. [10] showed that lymph node metastasis was identified at the time of initial diagnosis in $3 \%$ of tumors $<10$ $\mathrm{mm}, 66 \%$ in those 11 to $19 \mathrm{~mm}$, and $73 \%$ in those $\geq 20 \mathrm{~mm}$. Additionally, there have been many reports that lymph node metastasis increases depending on the size, and it is a common result that the lymph node metastases are rare in tumors less than 10 $\mathrm{mm}[6,11]$. However, the risk is not zero for tumors of $<10 \mathrm{~mm}$ and does not mean that there is little or no lymph node metastasis. Even in the case of 5 to $9 \mathrm{~mm}$ tumors, $8.4 \%$ of metastases were reported, and in some cases, metastases were reported even though the primary tumor was $4 \mathrm{~mm}$ [6]. In another study, multivariate analysis of patients with small rectal NETs $(\leq 10 \mathrm{~mm})$ found that venous invasion was the only factor independently associated with metastasis [12]. The depth of invasion is also correlated with lymph node metastasis, and Kasuga et al. [12] reported that the incidences of lymph node metastasis were $11.7 \%$ (limited to the mucosa or submucosa) and $87.5 \%$ (into or through the muscularis propria). In general, tumor differentiation and grade, as assessed by Ki-67 proliferation index or mitotic count are associated with more aggressive behavior and prognosis. However, small rectal NETs with lymph node metastasis are typically lowgrade well-differentiated tumors, and our case showed a similar pattern [13].

Rectal NETs in the lower rectum have the potential to metastasize to the LPLN along alternate lymphatic passages outside of the mesorectal envelope, similar to adenocarcinoma in the lower rectum. Colorectal cancer develops from the mucosal epithelium; on the other hand, colorectal NETs develop from Kultschitzky cells that are located in the deep mucosa. For this reason, lymph node metastasis appears to occur earlier in smaller sizes than in colorectal cancer, and especially, in tumors less than $10 \mathrm{~mm}$, LPLN metastasis appears to also accompany or recur. However, LPLN metastasis from rectal NETs is very rare, and there are very few reports written in the English language. Fujii et al. [14] summarized 8 and 4 reported cases of synchronous and metachronous LPLN metastasis from rectal NETs, respectively, and only 2 case reports were written in English. A total of 8 patients (66.6\%) showed no metastatic lymph nodes in the mesorectum and were identified as skip metastases, and 3 patients showed a primary tumor $\leq 10 \mathrm{~mm}$ in diameter. The tumor invasion depth was limited to the submucosa in 9 cases (75\%).

Due to their low-grade malignant potential and very slow growth, metastatic lateral pelvic lymph nodes are so small that preoperative identification with CT and magnetic resonance imaging may be difficult. Additionally, the indications for LPLD and how this approach contributes to patient prognosis are still unclear. Because of their rarity, there are no large population studies about only LPLD from rectal NETs, despite several reports on rectal NETs that had undergone radical resection with the indication of LPLD. In a study on rectal NETs that had undergone radical resection in 77 patients, 6 patients with a NET size of $7 \mathrm{~mm}$ or more underwent LPLD, and 5 of them were confirmed to have metastasis (6.5\%) [6]. In another study that performed LPLD with a lymph node size of $7 \mathrm{~mm}$ as an indication, LPLD was performed for 7 of a total of 102 patients. LPLNs were confirmed in 6 patients (5.9\%), representing the only LPLN metastases without distant metastasis in 4 of the 6 patients [15]. This study found that $66 \%$ of patients who had lateral lymph node metastasis had skip metastasis.

To date, there are no definite criteria for suspecting lymph node metastasis and no exact indication for LPLD in patients with rectal NETs. Due to a difference in origin, lymph node metastasis can occur even in small rectal NETs that do not have any other risk factors; thus, the initial radiological finding of lymph node metastasis is critical. Because about half of LPLN metastases were identified as skip lesions, even if there is no evidence of mesorectal lymph node metastasis, it is necessary to evaluate the status of LPLN through appropriate radiologic modality. Additionally, a study of the optimal cutoff size for LPLN metastasis should be conducted in the future. In small rectal NETs with LPLN metastasis, less aggressive behavior was observed, the recurrence interval was relatively long, and even metastatic lymph nodes did not grow rapidly. Therefore, surveillance with radiologic imaging over an extended period of time is needed. Also, radical resection including all metastatic lymph node metastases may contribute to a better prognosis, but considering the relatively favorable behavior, it seems necessary to investigate whether LPLD itself has a posi- 
tive effect on the patient's prognosis.

This case report shows that even small rectal NETs can be accompanied by extensive lymph node metastasis including LPLN similar to the tip of an iceberg. Initial evaluation and follow-up for lymph node metastases are important and although a longer follow-up period is required, performing radical resection with LPLD can be a curative option for improved patient prognosis.

\section{CONFLICT OF INTEREST}

No potential conflict of interest relevant to this article was reported.

\section{FUNDING}

None.

\section{REFERENCES}

1. Shah MH, Goldner WS, Benson AB, Bergsland E, Blaszkowsky LS, Brock P, et al. Neuroendocrine and adrenal tumors, version 2.2021, NCCN Clinical Practice Guidelines in Oncology. J Natl Compr Canc Netw 2021;19:839-68.

2. Mocellin S, Nitti D. Gastrointestinal carcinoid: epidemiological and survival evidence from a large population-based study $(n=25$ 531). Ann Oncol 2013;24:3040-4.

3. Gastrointestinal Pathology Study Group of Korean Society of Pathologists, Cho MY, Kim JM, Sohn JH, Kim MJ, Kim KM, et al. Current trends of the incidence and pathological diagnosis of gastroenteropancreatic neuroendocrine tumors (GEP-NETs) in Korea 2000-2009: multicenter study. Cancer Res Treat 2012;44: 157-65.

4. Dasari A, Shen C, Halperin D, Zhao B, Zhou S, Xu Y, et al. Trends in the incidence, prevalence, and survival outcomes in patients with neuroendocrine tumors in the United States. JAMA Oncol 2017;3:1335-42.

5. Zhao B, Hollandsworth HM, Lopez NE, Parry LA, Abbadessa B, Cosman BC, et al. Outcomes for a large cohort of patients with rectal neuroendocrine tumors: an analysis of the National Cancer Database. J Gastrointest Surg 2021;25:484-91.
6. Takatsu Y, Fukunaga Y, Nagasaki T, Akiyoshi T, Konishi T, Fujimoto $\mathrm{Y}$, et al. Short- and long-term outcomes of laparoscopic total mesenteric excision for neuroendocrine tumors of the rectum. Dis Colon Rectum 2017;60:284-9.

7. Maione F, Chini A, Milone M, Gennarelli N, Manigrasso M, Maione $\mathrm{R}$, et al. Diagnosis and management of rectal neuroendocrine tumors (NETs). Diagnostics (Basel) 2021;11:771.

8. National Comprehensive Cancer Network. Neuroendocrine and adrenal tumors (version 3.2021) [Internet]. Plymouth Meeting (PA): National Comprehensive Cancer Network; c2021 [cited 2021 Aug 13]. Available from: https://www.nccn.org/professionals/physician_gls/pdf/neuroendocrine.pdf.

9. Ramage JK, De Herder WW, Delle Fave G, Ferolla P, Ferone D, Ito $\mathrm{T}$, et al. ENETS consensus guidelines update for colorectal neuroendocrine neoplasms. Neuroendocrinology 2016;103:13943.

10. Gleeson FC, Levy MJ, Dozois EJ, Larson DW, Wong Kee Song LM, Boardman LA. Endoscopically identified well-differentiated rectal carcinoid tumors: impact of tumor size on the natural history and outcomes. Gastrointest Endosc 2014;80:144-51.

11. Al Natour RH, Saund MS, Sanchez VM, Whang EE, Sharma AM, Huang Q, et al. Tumor size and depth predict rate of lymph node metastasis in colon carcinoids and can be used to select patients for endoscopic resection. J Gastrointest Surg 2012;16:595-602.

12. Kasuga A, Chino A, Uragami N, Kishihara T, Igarashi M, Fujita R, et al. Treatment strategy for rectal carcinoids: a clinicopathological analysis of 229 cases at a single cancer institution. J Gastroenterol Hepatol 2012;27:1801-7.

13. Beppu N, Niki M, Kimura F, Matsubara N, Tomita N, Yanagi H, et al. A case of rectal carcinoid, $7 \mathrm{~mm}$ in diameter, with skip metastasis to the lateral lymph node. Mol Clin Oncol 2016;4:549-52.

14. Fujii Y, Kobayashi K, Kimura S, Uehara S, Miyai H, Takiguchi S. Indications for lateral lymph node dissection in patients with rectal neuroendocrine tumors: a case report and review of the literature. Mol Clin Oncol 2021;14:80.

15. Ushigome H, Fukunaga Y, Nagasaki T, Akiyoshi T, Konishi T, Fujimoto $\mathrm{Y}$, et al. Difficulty of predicting lymph node metastasis on $\mathrm{CT}$ in patients with rectal neuroendocrine tumors. PLoS One 2019;14:e211675. 